\title{
A catalogue of eclipsing variables ${ }^{\star} \star \star$
}

\author{
O. Yu. Malkov ${ }^{1,2}$, E. Oblak ${ }^{3}$, E. A. Snegireva ${ }^{4}$, and J. Torra ${ }^{2}$ \\ 1 Institute of Astronomy of the Russian Academy Sci. 48 Pyatnitskaya St., Moscow 119017, Russia \\ e-mail: malkov@inasan.ru \\ 2 Departament d'Astronomia i Meteorologia, Universitat de Barcelona, Avda. Diagonal 647, 08028 Barcelona, Spain \\ 3 UMR 6091, Observatoire de Besançon - CNRS, 41 bis Avenue de l'Observatoire, 25010 Besançon Cedex, France \\ ${ }^{4}$ Physical Department, Ural State University, Lenin Street 51, Ekaterinburg 620083, Russia
}

Received 26 March 2005 / Accepted 12 September 2005

\section{ABSTRACT}

A new catalogue of 6330 eclipsing variable stars is presented. The catalogue was developed from the General Catalogue of Variable Stars (GCVS) and its textual remarks by including recently published information about classification of 843 systems and making corresponding corrections of GCVS data. The catalogue ${ }^{1}$ represents the largest list of eclipsing binaries classified from observations.

Key words. binaries: eclipsing - catalogues

\section{Introduction}

Eclipsing binaries are very numerous. Independent stellar mass and luminosity determination is possible only for components of eclipsing binaries with the spectrum lines of the two components. However, they represent only some $5 \%$ of all known eclipsing binaries. So, for statistical investigations it is necessary to estimate at least approximate values of fundamental parameters (such as mass and radius) for eclipsing binaries with unknown spectroscopic elements.

This was a goal of early catalogues (Brancewicz \& Dworak 1980; Svechnikov \& Kuznetsova 1990). The process of mass and other astrophysical parameter estimation differs for different evolutionary classes of systems. So, to properly estimate astrophysical parameters one needs to know the class of a given system.

A procedure for determination of evolutionary class from observational data was first proposed by Svechnikov \& Snezhko (1974) and is still used in the General Catalogue of Variable Stars (1985-1988), hereafter GCVS. The procedure is based on a restricted number of systems with known classes contained in the old Svechnikov (1969) catalogue and, as our analysis shows, is not accurate enough. We have checked Svechnikov \& Snezhko's (1974) procedure for two classes of systems, detached main sequence and semi-detached. Stars from independent catalogues of detached main sequence

* Appendix A is only available in electronic form at http://www.edpsciences.org

$\star \star$ The catalogue is only available in electronic form at the CDS via anonymous ftp to cdsarc.u-strasbg.fr $(130.79 .128 .5)$ or via http://cdsweb.u-strasbg.fr/cgi-bin/qcat?]/A+A/446/785 systems by Svechnikov \& Perevozkina (1999) and Perevozkina \& Svechnikov (1999) and of semi-detached systems by Surkova \& Svechnikov (2005a,b) were classified according to the procedure using the GCVS data on depth of primary minimum, difference in depths of minima, period and spectrum. Altogether we evaluated 152 detached main sequence and 232 semi-detached systems. Only $57 \%$ and $70 \%$ of systems, respectively, were properly classified.

Useful ideas for classification of eclipsing binaries can be found also in a statistical study made by Giuricin et al. (1983). They mostly dealt with three classes of systems (detached, semi-detached and contact) and examined the statistics of the lightcurve morphologies, eclipse depths, periods and spectral types.

Thus, a more modern procedure is required that allows users to classify all eclipsing binaries based on a set of observational parameters even if the set is incomplete. The goal of the present study is to compile a catalogue of eclipsing variables extracting data for 6330 eclipsing binaries from the GCVS and its textual remarks. The catalogue will be used, in particular, to construct the procedure for classification of eclipsing binaries.

A literature search for evolutionary classes of the catalogued systems was made. A number of catalogues of eclipsing binaries of particular types has been recently prepared by the Ural group: Svechnikov \& Perevozkina (1999), Perevozkina \& Svechnikov (1999) for detached main sequence systems, Surkova \& Svechnikov (2005a), Surkova \& Svechnikov (2005b) for semi-detached systems, Dryomova et al. (2005) for near-contact binaries, etc. The majority of them were available in printed (Russian) version only, but 
standard descriptions for them are being prepared in the Centre for Astronomical Data (Moscow) and the catalogues will be included in the database of the Centre de Données Astronomiques de Strasbourg.

Data from other catalogues of eclipsing binaries were also used in the present investigation, e.g. Pribulla et al. (2003) for contact systems, Budding et al. (2004) for semi-detached systems, Shaw (1994) for near-contact binaries, etc. Altogether, 843 eclipsing systems of 9 different classes were classified.

The catalogue compilation procedure is described in Sect. 2. The description of the catalogue is also given. Some systems found to be mis-classified and/or have parameters other than the ones given in the GCVS. Corresponding corrections are made in our catalogue. The list of the systems is given in the Appendix.

\section{Catalogue of eclipsing variables}

In this section we describe the procedure of catalogue compilation, discuss various evolutionary classes of systems and present the format of the catalogue.

\subsection{Data from the GCVS}

All eclipsing binaries were chosen from the GCVS.

The following data, contained in the GCVS, are useful for evolutionary class determination: morphological type of the light curve (EA, EB or EW), maximum and minimum magnitudes, the photometric system for magnitudes, period of the variable, duration of primary eclipse, spectra of components.

Variability type, given in the GCVS for eclipsing variables (except morphological type of the light curve mentioned above) has not been used in this study, because it is based on the old Svechnikov \& Snezhko (1974) procedure.

Additional information was drawn from textual remarks in the GCVS and is tabulated as follows: secondary minimum magnitude, period variability indication, duration of secondary eclipse, duration of totality in primary and in secondary eclipses, phase of secondary eclipse.

We did not include in the catalogue three systems with unknown magnitude in maximum (X Phe) or minimum ( $\mathrm{T} \mathrm{Lac}$, BC Oct). So, the depth of primary minimum is given for all systems in the catalogue.

\subsection{Evolutionary classes}

The study supplies data with independently determined evolutionary classes of systems. The detached main sequence systems in the catalogue were classified according to two main sources, namely the catalogues of Svechnikov \& Perevozkina (1999) and Perevozkina \& Svechnikov (1999). For other sub-classes of detached systems, our main sources were Svechnikov (1969) catalogue, a study of Popper \& Ulrich (1977, detached systems with two subgiants) as well as lists of Popper (1980) and Malkov (1993). Catalogues of Surkova \& Svechnikov (2005a,b) served as main sources for semi-detached systems, while our sources for contact systems were catalogues of Shaw (1994), Pribulla et al. (2003) and Dryomova et al. (2005, near-contact systems).

We distinguish the following classes of eclipsing binaries: detached (D), semi-detached (S) and contact (C) systems. Detached and contact systems have sub-classes in our notation as listed below and we use the second (and in some cases the third) letter for the sub-class designation. We do not separate various sub-classes of semi-detached systems, but for uniformity use a one-letter identification ( $\mathrm{S}$ ) for a system class, reserving the second position for a potential sub-class designation.

\subsubsection{Detached systems}

Main sequence systems (DM) - detached main sequence systems, where both components are main sequence stars, which do not fill their inner Roche lobes.

Systems with a subgiant. A class of detached systems, where the secondary subgiant component does not fill its Roche lobe (so-called algols with undersized subgiant secondary or sd-d or after contact systems) was proposed by various authors and Svechnikov (1969) catalogue contains 17 such systems.

We have found that almost all of the catalogued subgiant systems were later re-classified to be semi-detached binaries, according to Surkova \& Svechnikov (2005a), Surkova \& Svechnikov (2005b) or Budding et al. (2004). They are: V0356 Sgr, AW Peg, $\lambda$ Tau, QY Aql, RY Gem, VW Cyg, S Cnc, TT Hya, RS Cep, RW Per, RX Gem, KO Aql, WW And and $\mathrm{Z}$ Ori.

The remaining systems are RZ Eri, BM Ori and $\alpha \mathrm{CrB}$. According to Popper (1980), RZ Eri belongs to classical RS CVn systems (DR in our notation, see below). BM Ori is a preMS system (Popper \& Plavec 1976) and its secondary of late A-type lies roughly $2^{\mathrm{m}}$ above the main sequence in the HR diagram. Tomkin \& Popper (1986) classify $\alpha \mathrm{CrB}$ as a detached main sequence system with the primary of spectral type A0, lying slightly above the zero age main sequence.

Thus, there is no confirmed detached system containing one subgiant. Such a stage of binary evolution is apparently too rapid to be easily observed. We eliminate the class of detached systems with a subgiant from further consideration.

Systems with two subgiants (DR) - detached systems with two subgiant components, where both stars do not fill their Roche lobe (alternative names: AR Lac systems, RS CVn systems, or long period RS CVn systems).

The hotter component in such binaries usually has a spectral type of either F or $\mathrm{G}$ and the systems show stronger emission lines in the spectrum outside eclipses. They typically are expected to have negligible mass exchange, although the majority of known RS CVn binaries are classified as detached in GCVS.

Giant and supergiant systems (DG) - systems with at least one member evolved well away from the main sequence (i.e., 
supergiant or late-type giant, e.g., VV Cep). Their evolutionary stage is generally unclear (Giuricin et al. 1983), but if the secondary component is a main sequence star, they are likely to be in a prior-to-mass exchange stage.

Pre-main-sequence (preMS) systems. There are a few binaries in the catalogue which are thought to be in a premain-sequence evolutionary stage (e.g., BM Ori). They are usually included in the sample of substantially unevolved detached systems with nearly main-sequence components (see, e.g., Giuricin et al. 1983). Due to their extreme paucity in the catalogue we also do not segregate them in a separate class, but consider them as DM systems.

\subsubsection{Semi-detached systems (S)}

Semi-detached systems (algols) are systems where the more massive component is the main sequence star and the less massive secondary component, which is usually the cooler and larger one, is a subgiant, which fills (or nearly fills) its Roche lobe.

Various sub-classes of semi-detached systems can be separated, although too few of the representatives of these sub-classes prevent the use of them for statistical analysis. Nevertheless, some special cases are discussed below.

Hot and cool algols. Popper (1980) in his comprehensive review on stellar masses distinguishes (somewhat arbitrarily), besides typical algols, two additional groups: hot semi-detached systems (in which the hotter component is an early B-type star and the cooler of type B or early A) and cool semi-detached systems of type $\mathrm{G}$ and later. The two groups consist of four systems each. Nelson \& Eggleton (2001) in their theoretical study also discuss hot and cool semi-detached systems.

Double-contact $(\beta$ Lyr) systems. According to Wilson (1979), both components in such a binary fill their Roche lobes exactly but do not touch each other due to asynchronous rotation of at least one of them. Svechnikov (1969) considers $\beta$ Lyr to be "on its way to a detached system with a subgiant". However, there is a doubt that $\beta$ Lyr can represent a sub-class, because more recent investigations (see, e.g. Linnel 2002) confirm the uniqueness of this system.

Undermassive (R CMa) systems. Both components in such systems are overluminous, oversized and hotter for their derived masses; neither component can be considered as a main sequence star (see Sarma et al.'s 1996, study of the prototype).

Systems in early stages of mass transfer. In some early-type detached systems the more massive component is evolving off the main sequence and almost filling its Roche lobe thereby starting mass transfer (see, e.g., Kallrath \& Strassmeier's 2000, study of BF Aur). Then a very rapid mass transfer takes place from the primary to the secondary, proceeding on a thermal time scale. Examples are TT Her (Milano et al. 1989b) classified as a contact system in the GCVS and AB Cru (Lorenz et al. 1994) classified as a detached system in the GCVS. The latter has already undergone a reversal of its mass ratio, so mass transfer from the (formerly more massive) secondary towards the primary is observed. In these cases a period change or/and a mass ratio reversal support the assumption that mass exchange has already started or is about to start.

Broken-contact systems. If the energy transfer in a close binary is insufficiently effective, a cycle consists of a long quiet phase in good contact and a short violent phase with rapid changes between contact, semi-detached and detached configurations (Kähler 2002). One such system observed in a semi-detached stage is CN And (Van Hamme et al. 2001). Broken-contact systems exhibit orbital periods in the range $0.3-0.7$ days and the spectral type is around $\mathrm{F}-\mathrm{G}$.

These groups consist of a relatively small number of binaries. That is why we do not separate these systems from other semi-detached pairs. Some of the stars from the latter two groups were classified as contact or detached binaries in GCVS

\subsubsection{Contact systems (C)}

According to Wilson's (1979) notation, they are in fact overcontact systems, where each component has a surface larger than its Roche lobe. They are synchronous circular orbit systems with common envelope. Besides contact (overcontact) systems in this section we consider also near-contact binaries.

Late-type systems (CW) - contact systems with spectrum of primary usually later than about F0 (called also W UMa systems).

According to Binnendijk (1977), CW (classical W UMatype contact) binaries were divided into A-type (the larger star is hotter, the primary minimum is the transit) and W-type (the smaller star is hotter, the primary minimum is the occultation). Hereafter we designate them CWA and CWW systems, respectively, and we leave the CW code for W UMa-type binaries of unknown sub-class.

Early-type systems (CE) - contact systems of early spectra with both components close to their Roche lobes. The spectrum of the more massive component is usually not later than about F0 (Svechnikov 1969) or even A0 (Pribulla et al. 2003).

Near-contact systems (CB). This class was introduced by Svechnikov (1969) as being similar to W UMa-stars, where both components do not fill their Roche lobes and their physical characteristics are similar to those of CW systems. They are also called short-period RS CVn-type or W UMa-similar noncontact systems. Formally they can be referred to as detached or semi-detached systems, but their lightcurves are peculiar and distorted by gaseous streams, and the brightness of the 
components is slightly variable, which makes them similar to contact systems of late spectral type. Pribulla et al. (2003) designate them as B-type contact systems (reminding $\beta$ Lyr lightcurve), whose components are in physical but not in thermal contact. These are also sometimes called binaries with two stars of very different effective temperature enclosed in a common envelope with the secondary located below and to the left of the main sequence in the HR diagram.

According to Shaw (1994) we distinguish F-type nearcontact binaries (the primary component is at or near the Roche lobe, the secondary component is inside the Roche lobe, the light curve is usually asymmetric) and V-type near-contact binaries (the primary component is inside the Roche lobe, the secondary component is at or near the Roche lobe, the light curve never shows asymmetry), respectively, CBF and CBV. $\mathrm{CB}$ is used for near-contact binaries of unknown sub-class.

We focus our attention on the following most numerous classes: DM, DR, DG, S, CW (CWA and CWW), CE, CB (CBF and $\mathrm{CBV}$ ). The other categories mentioned above are not considered separately in the present paper since only a few systems belong to these categories.

We also do not consider other evolutionary classes of close binary systems, namely: systems with a component under main sequence (hot subdwarf or WD), systems with a relativistic object, WR- and PN-systems as well as systems with novae and recurrent novae. Such classes were included by various authors, but they are not abundantly represented in the catalogue though some of such systems are included in the catalogue (in particular, DG class comprises some WR binaries). Systems of these classes can be found in the Catalogue of Evolved Close Binary Stars by Cherepashchuk et al. (1986).

Massive eclipsing systems with hot components (so called OB binaries) are often separated from other categories by various authors, when they discuss difficulties of their spectroscopic and photometric analysis (Popper 1980) or compare theoretical models and observed characteristics of stars (Figueiredo et al. 1994). We do not separate in the catalogue the massive systems. Instead they are distributed among different classes (DM, DG, S, CE) according to their evolutionary stages. An interested reader can consult a rather complete census of such systems recently prepared by Polushina (2004).

\subsection{Format of the catalogue}

The data in the catalogue are arranged in the following way.

1. Name of the star in alphabetical order of the constellation listing as in the GCVS.

2. Class of the system. This column contains various abbreviations as follows:

DM: Detached main sequence system;

DR: Detached subgiant system;

DG: Detached giant or supergiant system;

S: Semi-detached system;

C: Contact system of unknown sub-class;

CB: Near-contact system of unknown sub-class;

CBF: Near-contact F system;

CBV: Near-contact V system;
CE: Early-type contact system;

CW: Late-type contact system of unknown sub-class;

CWA: Late-type contact A system;

CWW: Late-type contact W system.

3. Alternative class (if the system is included in two published lists of stars of different classes).

4. Uncertainty flag (:) on evolutionary class

5. Morphological type of the light curve (EA, EB, EW; as in the GCVS).

Photometry

6. Magnitude at maximum brightness.

7. Depth of primary minimum, $A_{1}$, mag.

8. Depth of secondary minimum, $A_{2}$, mag.

9. The photometric system for magnitudes as in the GCVS.

\section{Period}

10. Period of the variable star, $P$, day.

11. Information on the sign of period variability as follows:

d: derivative is negative (period decreases).

i: derivative is positive (period increases);

$\mathbf{v}$ : derivative is non-zero and the sign varies (period increases and decreases);

u: derivative is non-zero, but the sign is unknown (period increases or/and decreases).

Eclipses (in phase $\times 1000$ unit)

12. Duration of primary eclipse, DI.

13. Duration of totality in primary eclipse, dI.

14. Duration of secondary eclipse, DII.

15. Duration of totality in secondary eclipse, dII.

16. Phase of secondary minimum, MinII-MinI.

\section{Spectra}

17. Spectral type of the primary star, $S p_{1}$.

18. Luminosity class of the primary star.

19. Spectral type of the secondary star, $S p_{2}$.

20. Luminosity class of the secondary star.

Acknowledgements. We thank Olga Durlevich for providing us with recent data from the General Catalogue of Variable Stars and Nikolaj Samus for his very useful comments on the GCVS. We acknowledge Galina Dryomova, Lyudmila Surkova and Tatyana Polushina, who provided us with their catalogues prior to publication. We thank Annemarie Bridges for her careful reading of and constructive comments on our paper. We are greatful to our referee Ronald W. Hilditch for helpful comments (particularly on the nature of V361 Lyr). O.M. thanks Maksim Tuchin for his support during the catalogue compilation. This work has been partially supported by the Spanish Ministerio de Ciencia y Tecnologia under contracts ESP 2001-4531-PE and ESP 2003-04352. This study was partly done while O.M. was an invited professor at the Université de Franche-Comté. This research has made use of the SIMBAD database, operated at the Centre de Données astronomiques de Strasbourg and of NASA's Astrophysics Data System Bibliographic Services.

\section{References}

Ahlin, P., \& Sundman, A. 1983, A\&A, 125, 391

Binnendijk, L. 1977, Vistas Astron., 21, 359

Brancewicz, H. K., \& Dworak, T. Z. 1980, Acta Astron., 30, 502

Budding, E., Erdem, A., Çiçek, C., et al. 2004, A\&A, 417, 263

Çakirli, O., Ibanoglu, C., Djurasevic, G., et al. 2003, A\&A, 405, 733 
Cherepashchuk, A., Katysheva, N., Khruzina, T., \& Shugarov, S. 1996, Catalog of Evolved Close Binary Stars (Brussel: Gordon and Breach Science Publishers), parts 1 and 2

Chochol, D., Pribulla, T., Rovithis-Livaniou, H., Rovithis, P., \& Kranidiotis, A. 1998, Contributions of the Astronomical Observatory Skalnate Pleso, 28, 51

Dryomova, G. N., Perevozkina, E. L., \& Svechnikov, M. A. 2005, A\&A, 437, 375

Erdem, A., Budding, E., Demircan, O., et al. 2005, AN, 326, 332

Faulkner, D. R., Samec, R. G., Hawkins, N. C., et al. 2004, A\&AS, 205,1804

Figueiredo, J., De Greve, J. P., \& Hilditch, R. W. 1994, A\&A, 283, 144

Frank, P., Kleikamp, W., \& Moschner, W. 1997, IBVS, 4481, 1

Giuricin, G., Mardirossian, F., \& Mezzetti, M. 1983, A\&AS, 54, 211

Hilditch, R. W., Collier Cameron, A., Hill, G., Bell, S. A., \& Harries, T. J. 1997, MNRAS, 291, 749

Hilditch, R. W., Bell, S. A., Hill, G., \& Harries, T. J. 1998, MNRAS, 296, 100

Houck, J. C., \& Pollock, J. T. 1986, PASP, 98, 461

Kähler, H. 2002, A\&A, 395, 907

Kallrath, J., \& Strassmeier, K. G. 2000, A\&A, 362, 673

Kholopov, P. N., Samus, N. N., Frolov, M. S., et al. 1985-1988, General Catalogue of Variable Stars, 4rd edn., Vols. I-III (Moscow, Nauka); Durlevich, O. V., Frolov, M. S., Kazarovets, E. V., \& Samus, N. N. 1994, The list of errors in the GCVS, 4th edn. I. Vols. I-III, Bull. Inf. CDS, 45, 19, see also http://www.sai.msu.su/groups/cluster/gcvs/gcvs/ for living edition

Kim, H.-I., Lee, J. W., Kim, C.-H., et al. 2004, PASP, 116, 931

Kjurkchieva, D. P., Marchev, D. V., Heckert, P. A., \& Ordway, J. I. 2005, AJ, 129, 1084

Lacy, C. H., \& Frueh, M. L. 1987, AJ, 94, 712

Linnel, A. P. 2002, MNRAS, 334, 963

Lipari, S. L., \& Sistero, R. F. 1986, MNRAS, 220, 883

Lorenz, R., Mayer, P., \& Drechsel, H. 1994, A\&A, 291, 185

Lorenz, R., Mayer, P., \& Drechsel, H. 1999, A\&A, 345, 531

Malkov, O. Yu. 1993, Bull. Inf. CDS, 42, 27

McFarlane, T. M., Hilditch, R. W., \& King, D. J. 1986, MNRAS, 218, 159

Milano, L., Barone, F., Mancuso, S., \& Russo, G. 1989a, Ap\&SS, 153, 273

Milano, L., Barone, F., Mancuso, S., Russo, G., \& Vittone, A. A. 1989b, A\&A, 210, 181

Nelson, C. A., \& Eggleton, P. P. 2001, ApJ, 552, 664

Niarchos, P. G., Hoffmann, M., \& Duerbeck, H. W. 1997, A\&AS, 124, 291

Perevozkina, E. L., \& Svechnikov, M. A. 1999, Catalogue of orbital elements, masses and luminosities of eclipsing variable stars (detached main-sequence type) with known elements of photometric orbit and unknown spectroscopic elements, Ekaterinbourg, Ural University, 122
Polushina, T. S. 2004, Astron. and Astrophys. Trans., 23, 213

Popper, D. M. 1980, ARA\&A, 18, 115

Popper, D. M. 1990, AJ, 100, 247

Popper, D. M. 1996, ApSS, 106, 133

Popper, D. M., \& Plavec, M. 1976, ApJ, 205, 462

Popper, D. M., \& Ulrich, R. K. 1977, ApJ, 212, L131

Pribulla, T., Kreiner, J. M., \& Tremko, J. 2003, Contributions of the Astronomical Observatory Skalnate Pleso, 33, 38

Pribulla, T., \& Vanko, M. 2002, Contributions of the Astronomical Observatory Skalnate Pleso, 32, 79

Samec, R. G., Carrigan, B. J., \& French, J. A. 1996, The Observatory, 116,365

Samec, R. G., Carrigan, B. J., Gray, J. D., et al. 1998, AJ, 116, 895

Sandage, A. 1993, AJ, 106, 703

Sarma, M. B. K., \& Vivekananda Rao, P. 1998, PASP, 110, 93

Sarma, M. B. K., Vivekananda Rao, P., \& Abhyankar, K. D. 1996, ApJ, 458, 371

Schilt, J. 1927, ApJ, 65, 124

Shaw, J. S. 1994, Mem. S. A. It., 65, 1

Smak, J. I. 1997, Acta Astron., 47, 345

Surkova, L. P., \& Svechnikov, M. A. 2005a, Catalogue of photometric, geometric and absolute elements of semi-detached eclipsing binary systems with known photometric and unknown spectroscopic orbits, in press

Surkova, L. P., \& Svechnikov, M. A. 2005b, Catalogue of photometric, geometric and absolute elements of semi-detached eclipsing binary systems with known spectroscopic orbits, in press

Svechnikov, M. A. 1969, Catalogue of orbital elements, masses and luminosities of close binary stars (Sverdlovsk: Publ. Ural University), Ser. Astronomia, issue 5, N 88

Svechnikov, M. A., \& Kuznetsova, Eh. F. 1990, Catalogue of approximate photometric and absolute elements of eclipsing variable stars, Vols. 1-2, Sverdlovsk, Ural University

Svechnikov, M. A., \& Perevozkina, E. L. 1999, Catalogue of orbital elements, masses and luminosities of eclipsing variable stars (detached main-sequence type) with known photometric and spectroscopic elements, Ekaterinbourg, Ural University, 5

Svechnikov, M. A., \& Snezhko, L. I. 1974, in Evidences of nonstationarity and stellar evolution, ed. A. A. Boyarchuk, \& Yu. N. Efremov (Moscow, Nauka), 181

Tomkin, J., \& Popper, D. M. 1986, AJ, 91, 1428

Torres, G., Andersen, J., Nordstrom, B., \& Latham, D. W. 2000, AJ, 119,1942

Van Hamme, W., Samec, R. G., Gothard, N. W., et al. 2001, AJ, 122, 3436

Vivekananda Rao, P., \& Radhika, P. 2002, Ap\&SS, 282, 103

Vivekananda Rao, P., Sarma, M. B. K., \& Prakash Rao, B. V. N. S. 1994, J. Astrophys. Astron., 15, 165

Wilson, R. E. 1979, ApJ, 234, 1054

Zola, S., Niarchos, P., Manimanis, V., \& Dapergolas, A. 2001, A\&A, 374,164 
O. Yu. Malkov et al.: Catalogue of eclipsing variables, Online Material $p 1$

\section{Online Material}




\section{Appendix A: The list of mis-classified systems and systems with parameters other than those given in the GCVS}

CX Aqr. Budding et al. (2004) list CX Aqr (erroneously classified as a CE system) among other semi-detached systems.

V694 Aql. The shortest period (0.450) S system in our catalogue V694 Aql was recently observed by Frank et al. (1997) who found that the period given in the GCVS was a spurious one. The new value is 0.82 .

AP Aur. Shaw (1994) includes AP Aur, erroneously classified as a CWA system, in his list of near-contact binaries (CB in our notation).

AR Boo. According to Houck \& Pollock (1986) the secondary depth of AR Boo is 0.7 (rather than $1^{\mathrm{m}}$ calculated from the GCVS data). Milano et al. (1989a) found AR Boo, erroneously classified as a CWA system, to have a large temperature difference between the components, so hereafter we consider AR Boo to be a CB system. This classification for AR Boo was recently confirmed by Faulkner et al. (2004).

SS Cam. As a comparison with Popper \& Ulrich's (1977) list shows, too early a spectrum is indicated in the GCVS for the secondary of SS Cam (correct value is K3).

RV CVn. Pribulla et al. (2003) indicate CB sub-class for RV CVn, the shortest period (0.270) CB system in our catalogue refering to the Schilt (1927) study. However, Schilt (1927) initially classified this star as a W UMa-type binary.

AK CMi. Contrary to the GCVS data for AK CMi Samec et al. (1998) report that their secondary eclipse occurs at phase 0.5 .

BH Cas. A new period of BH Cas is 0.406 (Zola et al. 2001) rather than 0.5 given in the GCVS.

V440 Cas. The EA type is given in the GCVS for CWW system V440 Cas, but as Samec et al. (1996) show, there are near-continuous changes in light in the out-of-eclipse portions of the (obviously EW type) light curve of V440 Cas.

V606 Cen. In their spectroscopic and photometric study of V606 Cen Lorenz et al. (1999) found the depth of primary and secondary minima for this system to be about 0.78 and $0.5 \mathrm{~V}$, respectively.

EG Cep. Chochol et al. (1998) have found EG Cep, erroneously classified as a CW system, to be a semi-detached system and its $A_{2}$ is somewhat smaller than indicated in the GCVS, $A_{2}=0.29 \mathrm{~V}$. According to Erdem et al. (1998) EG Cep appears to be a relatively uncommon Case A type evolving Algol system.

EI Cep. EI Cep was classified as a DR system, but according to Torres et al. (2000) it is a DM system.

YY Cet. Contrary to the GCVS data, period of YY Cet is 0.79 (McFarlane et al. 1986). They also found the system to be an Algol type binary rather than a near-contact system.

V Crt. The GCVS gives 0.57 for MinII-MinI of V Crt and Shaw (1994) classifies it as a doubtful CB system. However, according to a study of Sarma \& Vivekananda Rao (1998), it is a typical Algol system and its secondary minimum occurs exactly at phase 0.5 .
KU Cyg. KU Cyg, classified as a DG system, is actually a semi-detached system (Smak 1997).

V442 Cyg. Corrections in the GCVS photometric data were made for V442 Cyg (Lacy \& Frueh 1987).

V700 Cyg. According to Pribulla et al. (2003), incorrect spectral type is indicated in the GCVS for a CWW system V700 Cyg (should be G2V rather than F2).

V1073 Cyg. According to Pribulla et al. (2003), incorrect spectral type is indicated in the GCVS for a CWA system V1073 Cyg (should be F2V rather than A3Vm).

AX Dra. CW system AX Dra demonstrated unusually shallow secondary minima and we supposed it to be misclassified. In his Catalogue of Near-contact Binaries, Shaw (1994) marks AX Dra as "possible contact". Apparently, this assumption may be incorrect and AX Dra is a classical CB system. Indeed, Kim et al. (2004) have recently confirmed AX Dra to be a nearcontact binary.

RZ Eri. Contrary to the GCVS data for RZ Eri spectrum (A5+G8IV) Vivekananda Rao et al. (1994) have found it to be F0IV+G5-8III-IV.

BL Eri. According to Pribulla et al. (2003), the incorrect spectral type is indicated in the GCVS for a CWA system BL Eri (should be G0V rather than B5).

BM Eri. We have corrected the period for an unclassified system BM Eri. A value $P>20000^{\mathrm{d}}$ is mentioned in the textual remarks to the GCVS, but according to Ahlin \& Sundman (1983) the interval of the possible period is 9400-9490d.

PW Her. A relatively large value of DI-DII is given in the GCVS for a DR system PW Her (-0.12), but radial velocity and photometric data by Popper (1990) show a circular orbit for this system. As a comparison with Popper \& Ulrich's (1977) list shows, too late a spectrum is indicated in the GCVS for the primary of PW Her (correct value is G0).

V412 Her. Hilditch et al. (1998) found that the secondary component of V412 Her, erroneously classified as a CWA system, has a temperature excess of $50 \mathrm{~K}$ and this means that the system should be re-classified as a W-type W UMa system (CWW in our notation).

RT Lac. The EB type is given in the GCVS for DR system RT Lac (GCVS type EB), but it is possible to indicate the beginning and the end of eclipses on the light curves of RT Lac published by Çakirli et al. (2003), i.e., its light curve should be classified as an EA type one.

EM Lac. According to Pribulla et al. (2003), an incorrect spectral type is indicated in the GCVS for a CWW system EM Lac (should be G8V rather than F).

FT Lup. FT Lup was classified as a CBV system, but as Lipari \& Sistero (1986) show it is in fact a semi-detached system.

V361 Lyr. Hilditch et al. (1997) found that CE system V361 Lyr was actually a semi-detached system with the primary component filling its Roche lobe, and the secondary component well detached that corresponds to a CBF system in our notation. Its secondary minimum was found to be shallower than calculated from GCVS data. Hilditch et al. (1997) noted the gross asymmetry in the lightcurves during the first half of the system cycle and they omitted the data between phases 0.10 
and 0.48 to solve the light curves. So, we estimate $A_{2}$ for the system from their Fig. 1 to be about 0.4 .

VV Mon. As a comparison with Popper \& Ulrich's (1977) list shows, too late a spectrum is indicated in the GCVS for the primary of VV Mon (correct value is G2).

V508 Oph. According to Pribulla et al. (2003), incorrect spectral type is indicated in the GCVS for a CWA system V508 Oph (should be F9V rather than G5).

U Peg. According to Pribulla et al. (2003), incorrect spectral type is indicated in the GCVS for a CWW system U Peg (should be G2V rather than F3+F3). Corrections in the GCVS photometric data were made for U Peg according to Pribulla \& Vanko (2002).

XZ Per. The type of XZ Per, according to Popper (1996), is F2-5, earlier than that tabulated in the GCVS.

SZ Psc. As a comparison with Popper \& Ulrich's (1977) list shows, too late a spectrum is indicated in the GCVS for the primary of SZ Psc (correct value is F8).
UV Psc. It was found by Vivekananda Rao \& Radhika (2002) and recently confirmed by Kjurkchieva et al. (2005) that UV Psc previously classified as DM is a short period RS CVn system.

AQ Tuc. According to Pribulla et al. (2003), incorrect spectral type is indicated in the GCVS for a CWA system AQ Tuc (should be A9V rather than F3/5+A).

VV Vir. According to Sandage (1993) VV Vir is not an eclipsing variable, but belongs to RR Lyr stars.

AW Vir. According to Niarchos et al. (1997) AW Vir, erroneously classified as a CWA system, is a W-type W UMa system.

XZ Vul. The type of XZ Vul, according to Popper (1996), is F5-8, earlier than that tabulated in the GCVS.

AW Vul. Budding et al. (2004) list AW Vul (erroneously classified as a CE system) among other semi-detached systems. 